\title{
To the Editor of the Mathematical Gazette
}

DeAR SIR,- - Re "Going one better in geometric dissections" by H. Lindgren: In " Modern Puzzles " H. E. Dudeney No. 111 shows how to cut a Maltese cross into seven pieces which will form a square, (solution by A. E. Hill). This is one better than H. Lindgren's eight piece solution.

Re Maestro Puzzles:

The $6 \times 10$ rectangle has 2339 solutions as given in number code by the Univ. Manchester electronic computer (C. B. Haselgrove). These results have been translated into drawings by R. A. Fairbairn of Montreal.

The puzzle with the added square in central position has been solved by computer in 65 ways (Dana Scott, Princeton University). I had found 61 of these before seeing the computer results. With the square in positions other than the central one I have so far found and recorded over 7,000 solutions (without of course mirror images). There are strong indications that the final total will be about 10,000 .

Yours faithfully, Maurice J. Povah

\section{A HISTORIC COLLECTION OF MATHEMATICAL MODELS}

Recently, as a result of the sale of some property belonging to the Herschel family, there came to light a chest of drawers packed with mathematical models which were evidently made by Prof. A. S. Herschel about the year 1890 . The labels which accompany them indicate that some were exhibited at Munich in 1893 at an Exhibition sponsored by the Deutsche Mathematike Vereinigung; others were shown at the Royal Institution in 1897.

Most of the models are of wood and seem to represent types of crystal structure. They consist of cubes and other polyhedra strung on wire so as to show the changes produced by rotation and shear. Some of the cubes are covered with elaborate chess-board patterns painted in various colours. The meaning of these is not clear, but they may relate to contemporary ideas of molecular structure. There are models entitled "Full and Half repletions of Solid Space ", and others whose significance can only be surmised. There is a label, but unfortunately no models, entitled "Plaiting Process of making Crystal Models, devised and exhibited by John Gorham, M.R.C.S., Tonbridge, Kent". Presumably the models were returned to the exhibitor. Other oddities include a Mobius strip made from part of a fashion plate, a linkage of kites and rhombi in a match-box, and a square sheet of paper elaborately folded into a plane octagon with folds ingeniously tucked. If any reader is interested in this collection of models, he can obtain particulars from Miss W. A. Cooke, 6 Cherry Orchard, Stoke Poges, Bucks, to whom they now belong.

\section{WANTED}

Hobson, E. W., Theory of Spherical and Ellipsoidal Harmonics (C.U.P. 1931). Please write to the Librarian, Rutherford College of Technology, Newcastle upon Tyne, 1. 